\title{
Continuation and Automation in Spinning
}

\author{
By Shoichi Ishikawa, Member, TMSJ \\ Tokyo Institute of Technology, Tokyo
}

\section{Foreword}

In manufacturing industries, the final goal that everyone strives for is to make a completely automatic machinery that takes in raw material on its one end and delivers finished product from the other end.

Although the cotton spinning is among the relatively modernized industries, it comprises of numerous processing stages making it far from the final goal mentioned above.

But, recently, with the advent of high speed and automatic processing machines a continuous and automatic production became feasible by connecting these machines in series.

On the other hand, the rapid development of Japanese industries brought about a shortage in labor and a rapid rise in wages.

This has been forcing the Japanese textile industry to change from a labour intensive industry to a capital intensive industry.

As one evidence of this situation, the Toyobo Co. in 1960 announced its completion of a pilot plant under the CAS system.

Then, in 1962, the same company built 30,000 spindles of CAS equipments in its Hamamatsu mill for a commercial production. It certainly was an epoch-making event. Following Toyobo, many similar systems have been developed by many companies in Japan and in other countries. It is going to start for an age of continuous spinning method.

We discuss here some of the continuous spinning systems developed in Japan.

Continuous automated spinning systems can be classified by the following characteristics :

a) Types of spinning process

b) Types of machines used

c) How processes are connected

Since each of the three items above contain many varieties, the conbination of the three provides a large number of alternatives.

In a continuous and automatic spinning, each component machine must have a high-productivity, a very low down time and must withstand dust and con- tinuous running.

Cleaning of macines not only influences yarn qualities, increases end breaks and induces machines damages but also increases labour requirements.

In TAS (Toyota), an emphasis is placed on the cleaning of machines by calling it "Cleaning automation".

After the drawing process transportation of products from one process to another is a big factor in labor requirement.

In KATS (Kurabo), on account of this, places emphasis on transportation and calls it "Auto-Transfer Spinning".

\section{Classification of Continuous Spinning by Process Types}

By processes, continuous automated spinning systems are classified into following types.

(1) Combed yarns.........KMS-1 (Kurehabo)

(2) Carded yarns

a) Systems not using speed frames.......CAS (Toyobo), NASS (Nittobo), and TNS (Tuzukibo)

b) Systems using speed frames ...... DASS (Daiwabo), TAS (Toyota) and KATS (Kurabo)

It is quite understandable that the more numerous carded yarn process first becomes the object of continuation and automation.

But it is also of importance to develop a continuous automated system for combed yarns which are the high-quality yarns.

Out-line of KMS-1 is as follows.

Connection of blowing machine and card is carried out by the NCF equipment which is similar to that used in CAS.

Heavy card slivers are produced at a low speed.

Slivers from 36 cards are gathered on the conveyer and fed to the drawing lap machine.

Slivers from 6 deliveries of drawing lap machine run on a table to the lap winder equipped with an automatic doffer to form a comber lap. 


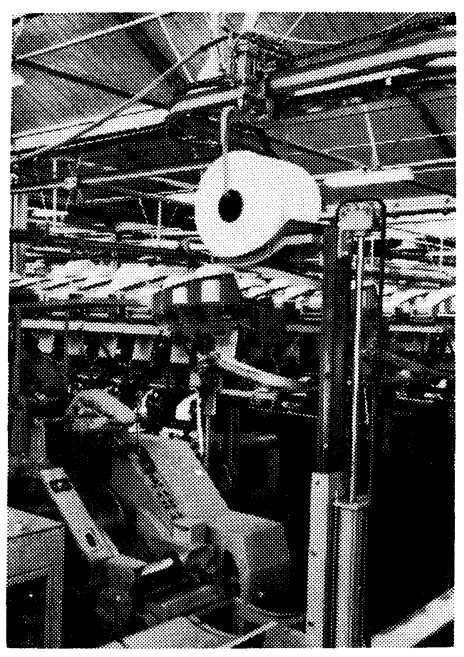

Fig. 1 KMS, Automatic Lap Transporter

Laps are carried by the automatic lap transporter and fed to combers by an automatic lap feeder.

The combed slivers from 3 combers equipped with pre-draft device are gathered and fed to the high-speed drawing frame having a thickness control device.

These processes are followed by conventional roving, spinning frame and auto-winder. The spinning frames are tended with newly developed auto-copdoffers.

In Japan, several companies have developed and used the super high draft spinning frames for shortening the processing stages.

In the early stage of the development of continuous spinning these super high draft frames were used. Later, however, continuous and automatic systems incorporating roving frames have been developed, because in Japan most of the spinning frames are not of super-high draft types and because recent speed frames have been greatly improved.

In Japan research and development have been under way on the open end spinning systems. The open end systems not only mean speed up of spinning but also frame eliminates winding machine and roving frames resulting in a great labor saving. They are hoped useful for the continuation and automation of the entire spinning industry.

Machines used in the continuous and automatic systems and their connecting methods are as follows.

\section{Blowing Machinery}

In recent years one process pickers are in wide uses. The fast developing bale pluckers are effective in saving labour, and also function as cotton blenders and cleaners.

In DASS, D type plucking blender is developed. This machine produces cotton laps of controlled density by each type of cotton, and lay the laps automatically placing one lap upon another in a sandwich form to feed the next processing stage.

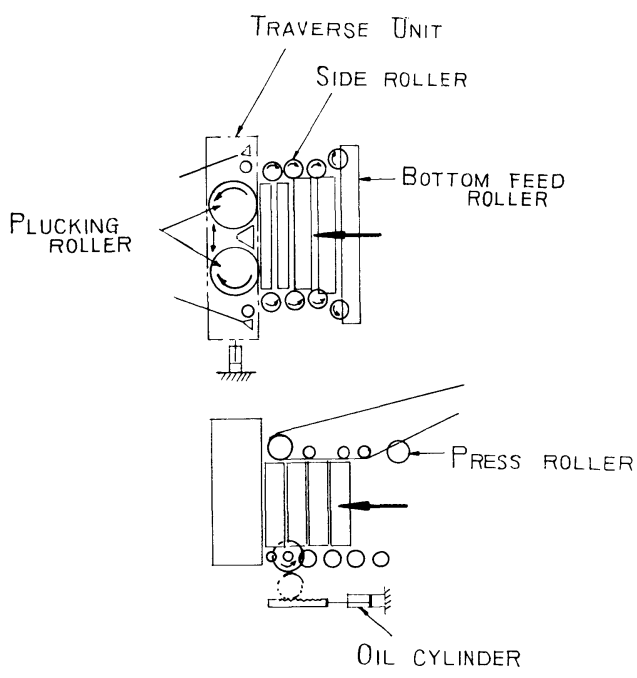

Fig. 2 KATS, Vertical Plucker

In KATS, a vertical plucker is used. It plucks horizontaly the side of a cotton bale.

The blowing unit carries opened cotton in loose form by air to the condenser hopper without forming laps. The condenser hopper, thereafter, feeds unit hopper placed at the end of each line of cards.

\section{Cards}

In CAS, NASS, KMS and KATS, cotton from unit hopper is fed evenly to reserve boxes (chute). A reserve box is a thin vertical chute equipped to each card.

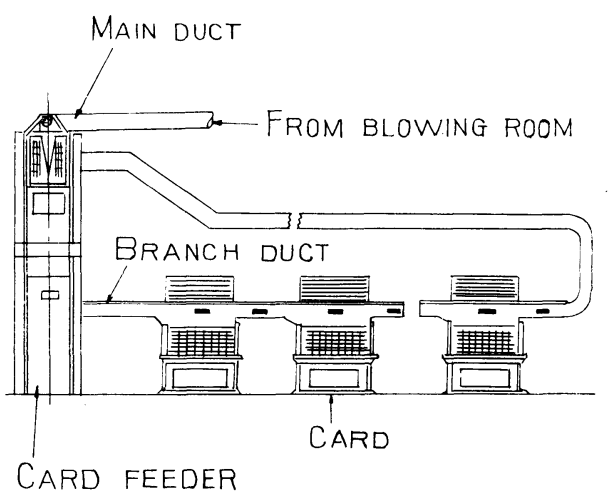

Fig. 3 Dass Cards 
In DASS and TAS, such chute is not used. In DASS, cotton carried by air is fed to a filter conveyer which is equipped on each card and forms the loose fiber in a sheet.

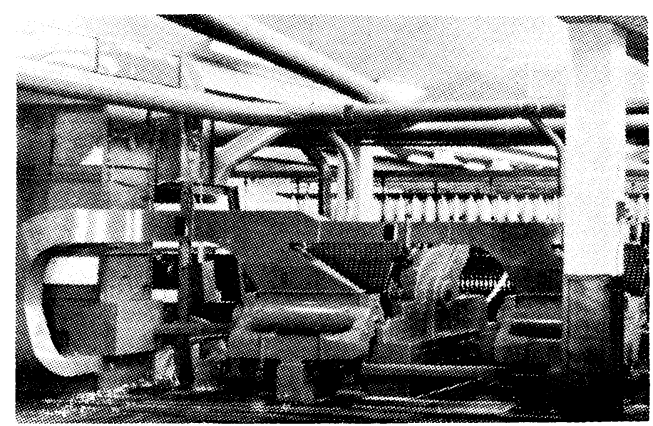

Fig. 4 TASS Cards

In TAS, cotton is evenly fed by an intermittent air stream on a feed cage equipped to each card. An overflow of cotton does not occur in this system.

CAS uses 6 to 8 multi cards each comprising of 4 cards since a high production card was not developed at the time of its development and in DASS and KA TS, D type and OS I type cards developed already by each company are used.

Recently, with the advent of high production cards, 3 cards are used as a unit in TAS, and 4 cards in TNS.

In every case, dust and fly are collected automatically. Slivers made from a line of cards are carried by belt conveyer, are doubled and fed to drawing frames.

In DASS, slivers are formed into $10 \mathrm{~cm}$ width ribbons for more effective blending and drafting.

In these continuous systems speeds of cards, conveyers and drawing frames, etc. should not differ by more than a few percent even during the acceleration and deceleration in start up and shut down in order to maintain a normal sliver quality.

In CAS, cards, conveyors and drawing frames are operated synchronously by a simplified selsyn.

In NASS, effect of speed difference during start up and shut down of cards are absorbed by placing a reserve pan with a micro switch equipped to the delivery end of each card.

In TAS, cards also have sliver reserve box and are controlled by a card controller on one of the cards.

In DASS, a group of cards are driven by one motor, and a drive shaft extending from the card doffer drives the sliver conveyor and the drawing frame to obtain equal machine speed:

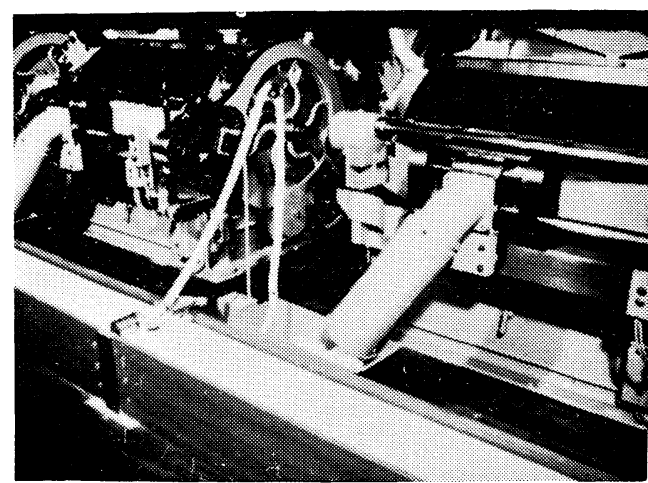

Fig. 5 NASS Cards

\section{Drawing Frames}

Continuous spinning systems incorporate highspeed drawing frames that has been developed in recent years. In CAS, drawing speed is $130-150 \mathrm{~m} / \mathrm{min}$. In TAS (also KATS), drawing speed is 200-250 $\mathrm{m} / \mathrm{min}$. Both drawing frames have sliver thickness controllers.

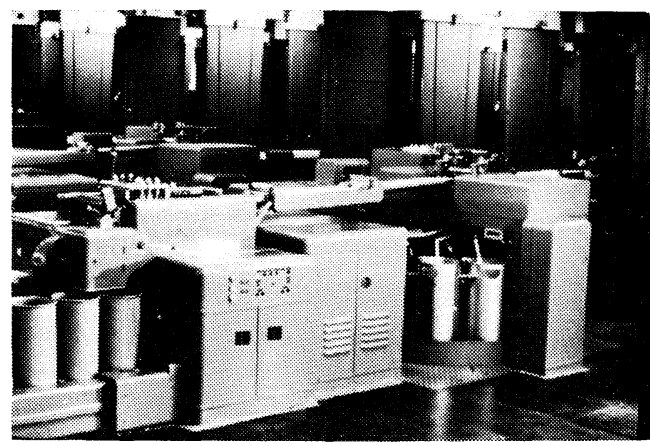

Fig. 6 CAS Drawing Frame

In DASS and TAS, the sliver thickness controllers are closed loop systems using air micrometers. In CAS, NASS and KMS, open loop control systems are used.

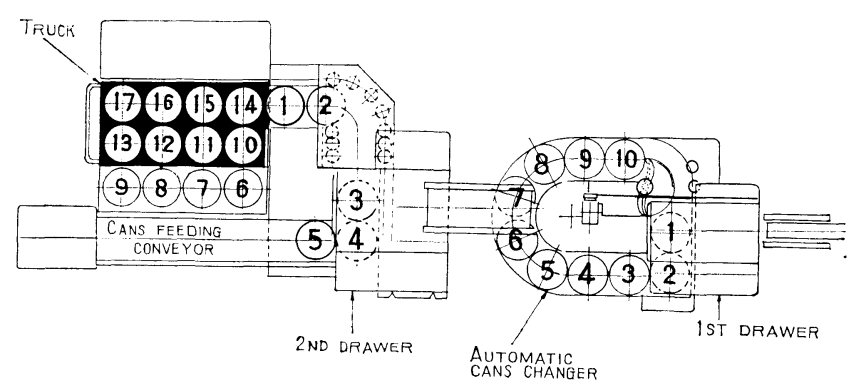

Fig. 7 TAS Drawing 
DASS has just one drawing passage. Whereas CAS, NASS and TAS have two drawing passages. In the latter cases, slivers from the first drawing frames fill cans, and these slivers are doubled and fed to the second drawing frames.

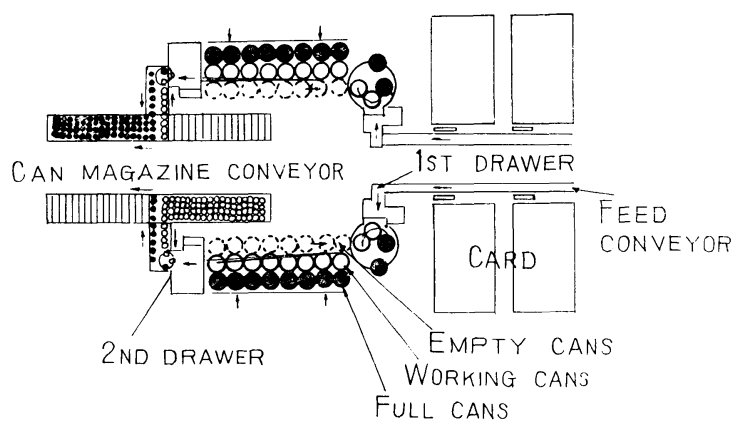

Fig. 8 NASS Drawing

In CAS sliver cans are placed to form a carrousel, in TAS the cans are arranged in a field track form, and in NASS icans are placed in three rows the first row being full cans, the second row working cans and the third row empty cans. In each cases cans are used repeatedly; exchange of cans and feeding of sliver are done automatically.

Full cans are stacked on a cans carrier in two stories in CAS, stored on a can magazine conveyer in NASS, put automatically on a can carrier in TAS.

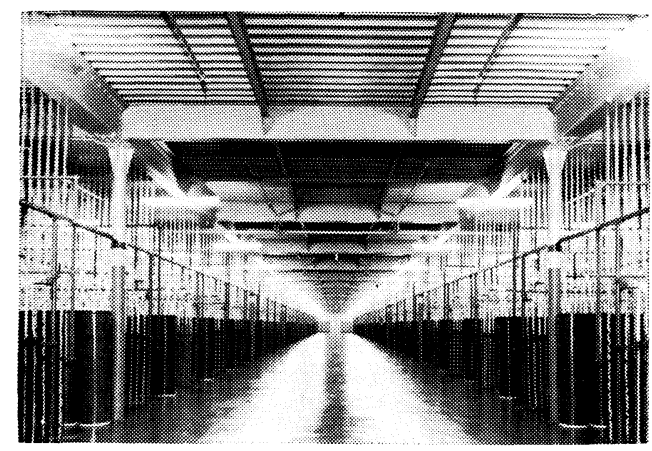

Fig. 9 TNS Cans Room

In TNS, the mill is a three story building, and drawing slivers are aged in a conditioning rooms on the second and third stories.

Then, each sliver is fed through vinyl-chloride pipe to each spindle of spinning frame on the first story.

\section{Speed Frames}

In TAS, the can carrier are carried to the back of a speed frame and slivers are fed directly from cans on the carrier.

In KATS, speed frames are placed at a particular relative position to the drawing frames. Full cans at the drawing frames are carried to the speed frames on an automatic can conveyor. When emptied the cans are pushed out to ride on a can returning conveyer which carries the empty cans automatically back to the drawing frames.

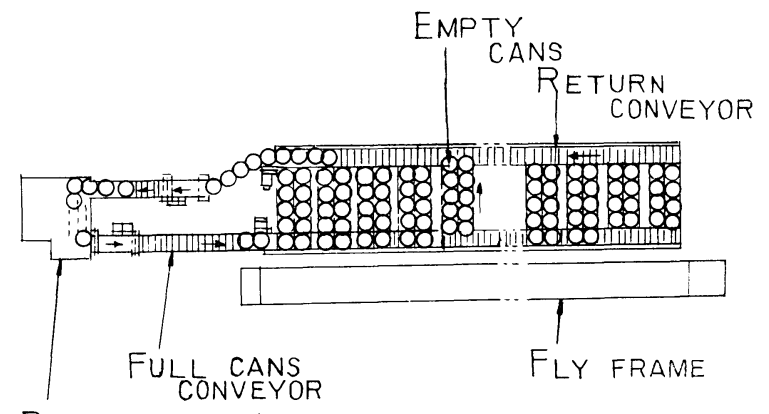

DRAWING FRAME

Fig. 10 KATS, Automatic Cans Transporter

For continuous spinning systems new simplex fly frame have been developed. The D-L type frame in DASS is a 4 line double apron frame. Its flyer's leg different from conventional one reaches to down part. Its package is $500 \times 150 \mathrm{~mm}$; speed is
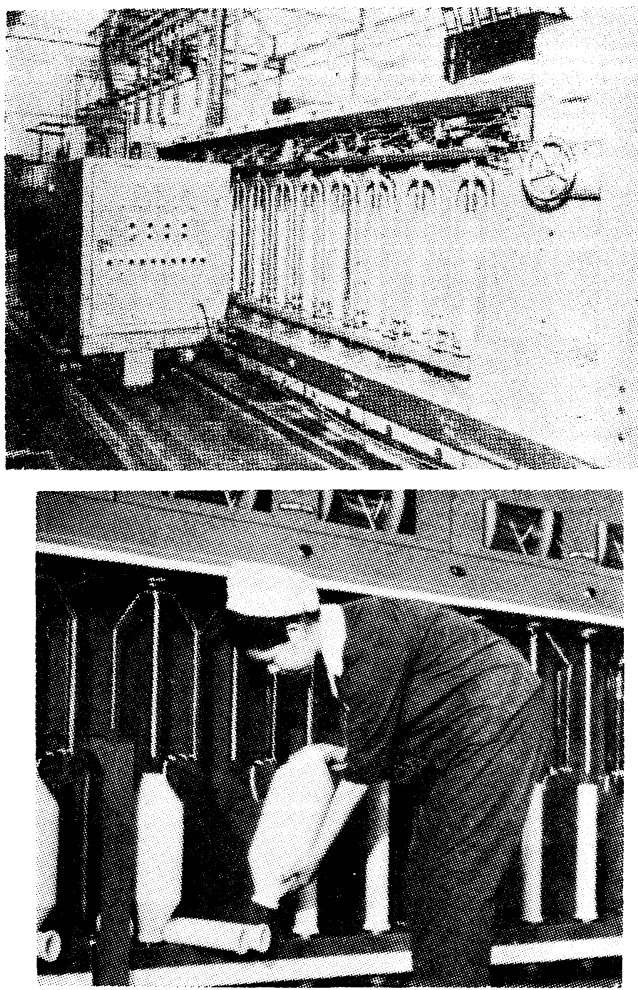

Fig. 11 DASS, Fly Frame and Automatic Roving Doffer 

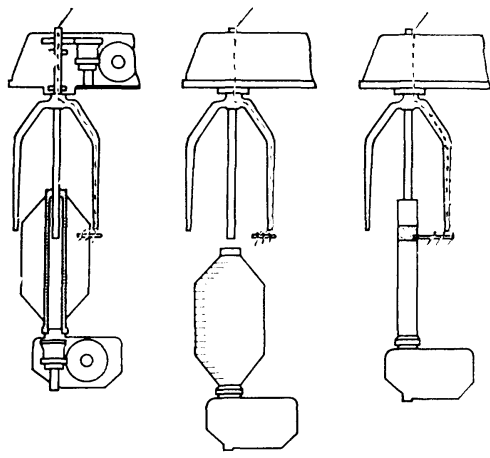

Fig. 12 TAS Fly Frame

1,300 r.p.m. The speed frame is tended with an automatic roving doffer.

The FXA roving frame of TAS has an over hung type flyer, with a large package size of $335 \times 175 \mathrm{~mm}$ (14/7 in.). Its speed is 1,200 r.p.m. Its doffing is easy requiring only one-third the time for a conventional frame.

In DASS, rovings are carried to the spinning frame by an automatic bobbin carrier that operates in synchronization with the auto roving doffer. When the carrier returns it carries empty bobbins from the spinning frame to the speed frame.

KATS also uses automatic roving carriers.

\section{Spinning Frames}

In CAS, NASS and TNS, super high draft spinning frames are used. In CAS, sliver cans are put between spinning frames. 40's yarns are spun at a high draft ratio of 360 by means of proper draft parts and drafting conditions. In NASS, because small sliver cans are placed on top of the spinning frames, the Audomac doffer is used both for the purpose of changing cans and for doffing bobbins.

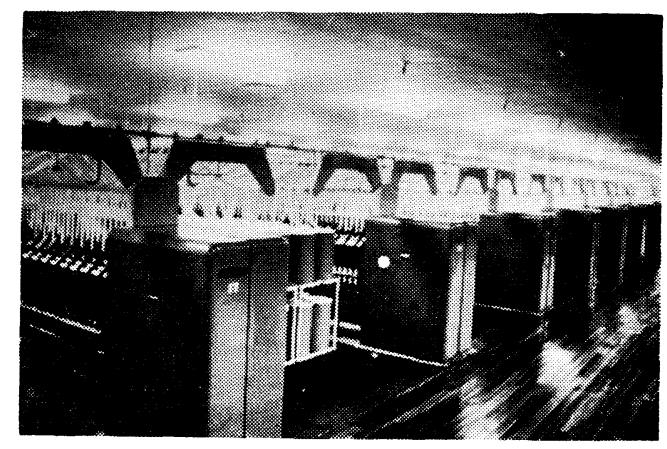

Fig. 13
Automatic cop doffers are classified into single cop doffing, group cop doffing and all cop doffing system. In TAS, the doffing system is single cop doffing. In CAS, DAS and KMS, it is group cop doffing.

In KMS, the automatic doffer is made thin so that the doffer can work in the narrow space between spinning frames and pillars in a conventional mill.

The automatic doffer of NASS has a loading station. The doffer automatically inserts full cops onto pegs and lift up empty bobbins. Full cops are carried to the winder on a conveyer.

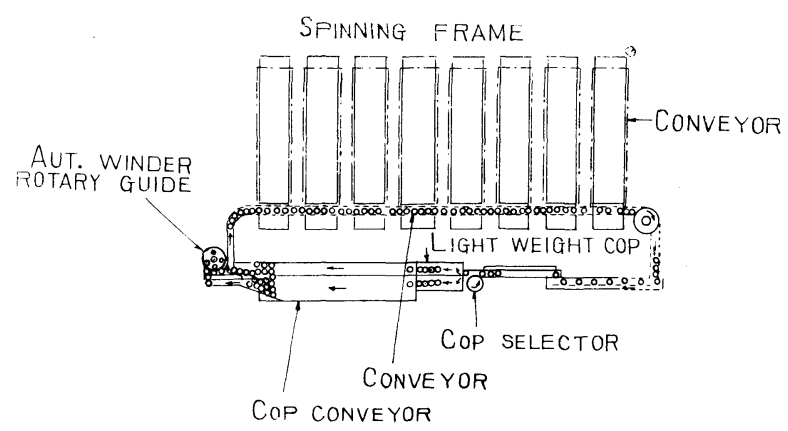

In KATS, transportation from the spinning frames to the winding machines is automatic. Doffed cops are pulled by magnets on a conveyer attached to a spinning frame. The automatic cop feeder receive cops from the conveyer attached to the spinning frame, and kicks out cops of light weight by means of an automtic cop selector. Then, cops are fed to the automatic winder by a conveyer. These devices are controlled from the central control panel. Empty bobbins from the automatic winder are automatically selected and put into boyes.

\section{Winders}

Continuous spinning systems use automatic high speed winders developed recently. Some of these winders are equipped with autocop feeders that feed cops automatically to the winder, or a cop selector (for doffing constant weight chease), or an empty bobbin carrier (including those capable of handling bobbins with remaining yarns).

\section{Continuous and Automatic Systems in Worsted Spinning}

Such methods as feeding loose fiber to the card, carrying card slivers on a conveyor to the next ma- 
chine and the use of sliver thickness controller on a gill box, are originally developed in worsted spinning.

It is fair to say that these methods originated in worsted spinning have been used to good advantage in developing automatic and continuous cotton spinning. However, because the processes are complex in worsted spinning and product lots are small, it is difficult to develop a continuous system.

With the advent of the high speed gill boxes, the chain driven gill boxes and the sliver thickness con- trollers, partially continuous processes have been developed by several companies.

Last year, Okuma Iron Co. disclosed PD-PD type Automatic can feeder for shortening of processes in the top and drawing processes. This feeder doffs filled cans automatically in the drawing process, and exchanges automatically the doffed cans with empty cans that fed the next process and at the same time ties the two ends of slivers automatically using the principle of the needle punch. 